Supplement of Arch. Anim. Breed., 63, 53-59, 2020

https://doi.org/10.5194/aab-63-53-2020-supplement

(C) Author(s) 2020. This work is distributed under

the Creative Commons Attribution 4.0 License.

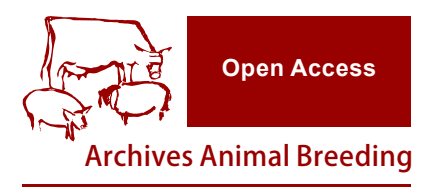

(c) (i)

Supplement of

\title{
Assessment of genetic diversity in main local sheep breeds from Romania using microsatellite markers
}

Andreea Dudu et al.

Correspondence to: Sergiu E. Georgescu (georgescu_se@yahoo.com, sergiu.georgescu@bio.unibuc.ro)

The copyright of individual parts of the supplement might differ from the CC BY 4.0 License. 
Table S1. The characteristics of the microsatellite markers used in the current study.

\begin{tabular}{|c|c|c|c|c|}
\hline Locus & Primer sequence & $\begin{array}{l}\text { GenBank } \\
\text { accession number }\end{array}$ & $\begin{array}{l}\text { Allelic } \\
\text { (bp) }\end{array}$ & range \\
\hline OarCP20 & $\begin{array}{l}\text { F: ggcatttcatggctttagcagg } \\
\text { R: gtttgatcccctggaggaggaaacgg }\end{array}$ & L01531 & $70-110$ & \\
\hline OarCP34 & $\begin{array}{l}\text { F: gctgaacaatgtgatatgttcagg } \\
\text { R: gggacaatactgtcttagatgctgc }\end{array}$ & U15699 & $102-120$ & \\
\hline OarCP49 & $\begin{array}{l}\text { F: cagacacggcttagcaactaaacgc } \\
\text { R: gtggggatgaatattccttcataagg }\end{array}$ & U15702 & $76-140$ & \\
\hline MAF70 & $\begin{array}{l}\text { F: cacggagtcacaaagagtcagacc } \\
\text { R: gcaggactctacggggectttgc }\end{array}$ & M77199 & $124-160$ & \\
\hline MAF65 & $\begin{array}{l}\text { F: aaaggccagagtatgcaattaggag } \\
\text { R: ccactcctcctgagaatataacatg }\end{array}$ & M67437 & $120-144$ & \\
\hline MAF33 & $\begin{array}{l}\text { F: gatctttgtttcaatctattccaatttc } \\
\text { R: gatcatctgagtgtgagtatatacag }\end{array}$ & M77200 & $118-166$ & \\
\hline MAF35 & $\begin{array}{l}\text { F: agttacaaatgcaagcatcatacctg } \\
\text { R: tcaagaattttggagcacaattctgg }\end{array}$ & M80518 & $104-124$ & \\
\hline MAF214 & $\begin{array}{l}\text { F: aatgcaggagatctgaggcagggacg } \\
\text { R: gggtgatcttagggaggttttggagg }\end{array}$ & M88160 & $186-236$ & \\
\hline BM143 & $\begin{array}{l}\text { F:acctgggaagcctccatatc } \\
\text { R: ctgcaggcagattctttatcg }\end{array}$ & AF394448 & $98-118$ & \\
\hline BM1314 & $\begin{array}{l}\text { F: ttcctcctcttctctccaaac } \\
\text { R:atctcaaacgccagtgtgg }\end{array}$ & G18433 & $136-179$ & \\
\hline HSC & $\begin{array}{l}\text { F: ctgccaatgcagagacacaaga } \\
\text { R:gtctgtctcctgtcttgtcatc }\end{array}$ & M90759 & $265-295$ & \\
\hline McM42 & $\begin{array}{l}\text { F: catctttcaaaagaactccgaaagtg } \\
\text { R: cttggaatccttcctaactttcgg }\end{array}$ & L34281 & $73-103$ & \\
\hline McM527 & $\begin{array}{l}\text { F: gtccattgcctcaaatcaaattc } \\
\text { R: aaaccacttgactactccccaa }\end{array}$ & L34277 & $163-181$ & \\
\hline OarFCB20 & $\begin{array}{l}\text { F: ggaaaacccccatatatacctatac } \\
\text { R: aaatgtgtttaagattccatacatgtg }\end{array}$ & L20004 & $80-116$ & \\
\hline OarFCB11 & $\begin{array}{l}\text { F: gcaagcaggttctttaccactagcacc } \\
\text { R:ggcctgaactcacaagttgatatatctatcac }\end{array}$ & L01531 & $120-150$ & \\
\hline INRA063 & $\begin{array}{l}\text { F: atttgcacaagctaaatctaacc } \\
\text { R: aaaccacagaaatgcttggaag }\end{array}$ & X71507 & $155-211$ & \\
\hline INRA127 & $\begin{array}{l}\text { F:ctacagctctgatgagaacc } \\
\text { R: cgttttctcaaacttcattgcc }\end{array}$ & X71550 & $181-213$ & \\
\hline TGLA53 & $\begin{array}{l}\text { F: gctttcagaaatagtttgcattca } \\
\text { R: atcttcacatgatattacagcaga }\end{array}$ & - & $126-148$ & \\
\hline
\end{tabular}

